\title{
Effective prevention of sorafenib-induced hand-foot syndrome by dried-bonito broth
}

This article was published in the following Dove Press journal: Cancer Management and Research

\begin{abstract}
Kenya Kamimura,' Yoko Shinagawa-Kobayashi,' Ryo Goto,' Kohei Ogawa,' Takeshi Yokoo,' Akira Sakamaki,' Satoshi Abe,' Hiroteru Kamimura,' Takeshi Suda, ${ }^{2}$ Hiroshi Baba, ${ }^{3}$ Takayuki Tanaka, ${ }^{4}$ Yoshizu Nozawa, ${ }^{5}$ Naoto Koyama, 6 Masaaki Takamura,' Hirokazu Kawai,' Satoshi Yamagiwa,' Yutaka Aoyagi,' Shuji Terai'

'Division of Gastroenterology and Hepatology, Graduate School of Medical and Dental Sciences, Niigata University, Niigata, Niigata, Japan; 2Department of Gastroenterology and Hepatology, Uonuma Institute of Community Medicine, Niigata Medical and Dental Hospital, MinamiUonuma, Niigata, Japan; ${ }^{3}$ Division of Anesthesiology, Graduate School of Medical and Dental Sciences, Niigata University, Niigata, Niigata, Japan; ${ }^{4}$ Uonuma Eye Clinic, Uonuma, Niigata, Japan; ${ }^{5}$ Institute of Food Sciences and Technologies, Ajinomoto Co., Inc., Kawasaki, Kanagawa, Japan; ${ }^{6}$ Institute for Innovation, Ajinomoto Co., Inc. Kawasaki, Kanagawa, Japan
\end{abstract}

Correspondence: Kenya Kamimura Division of Gastroenterology and Hepatology, Graduate School of Medical and Dental Sciences, Niigata University, I-757 Asahimachi-dori, Chuo-ku, Niigata, Niigata 9518510 , Japan

Tel +8I 252272207

Fax +8I 252270776

Email kenya-k@med.niigata-u.ac.jp
Background: Sorafenib (SOR) is a molecular medicine that prolongs the survival of patients with hepatocellular carcinoma (HCC). Therefore, the management of side effects is essential for the longer period of continuous medication. Among the various side effects, hand-foot syndrome (HFS) is the most common, occurring in $30 \%-50 \%$ of patients, and often results in discontinuation of the SOR medication. However, its mechanism has not been clarified, and no effective prevention method has been reported for the symptoms. Therefore, this study aimed to analyze its mechanism and to develop an effective prevention regimen for the symptoms.

Materials and methods: To assess the mechanism of SOR-induced HFS, the peripheral blood flow in the hand and foot was carefully monitored by Doppler ultrasound, thermography, and laser speckle flowgraphy in the cases treated with SOR and its contribution was assessed. Then, the effect of dried-bonito broth (DBB), which was reported to improve peripheral blood flow, on the prevention of the symptom was examined by monitoring its occurrence and the peripheral blood flow.

Results: A total of 25 patients were enrolled in this study. In all, eight patients developed HFS, and all cases showed a significant decrease in the peripheral blood flow. DBB contributed to an increase in the flow $(p=0.009)$ and significantly decreased occurrence of HFS $(p=0.005)$ than control. Multivariable analysis showed that the ingestion of DBB is a significant independent contributor to HFS-free survival period ( $p=0.035$ ).

Conclusion: The mechanism of SOR-induced HFS involves a decrease in the peripheral blood flow, and the ingestion of DBB effectively prevents the development of the syndrome by maintaining the flow.

Keywords: sorafenib, hepatocellular carcinoma, hand-foot syndrome, blood flow, bonito broth

\section{Introduction}

Sorafenib (SOR) is a molecular medicine for advanced hepatocellular carcinoma (HCC) cases unresponsive to locoregional treatments, such as catheter-based chemotherapy and embolization, as well as cases with extrahepatic metastatic lesions. SOR is expected to prolong survival in patients with $\mathrm{HCC} ; ;^{1-3}$ therefore, the management of side effects to prolong the use of medication is essential for controlling the disease and maintaining the quality of life (QoL) of the patients. Various side effects have been reported for SOR administration, including hand-foot syndrome (HFS), which is reported in $30 \%-50 \%$ of patients. ${ }^{4-6} \mathrm{HFS}$ is a side effect often seen in the patients treated with chemotherapy agents, such as fluoropyrimidines, and kinase inhibitors. ${ }^{5,78} \mathrm{HFS}$ generally occurs after 2-4 weeks of treatment, causing redness, pigmentation, keratinocytosis, phlyctenule, 
and nail deformity, especially on the skin of the hands and feet, which causes sensory disturbances, numbness, and pain. ${ }^{7}$ These symptoms significantly affect the QoL of the patients, and in some cases, they need to discontinue treatment, which may lead to disease progression. Since SOR is a tyrosine kinase inhibitor affecting vascular endothelial growth factor, vascular endothelial growth factor receptor, and platelet-derived growth factor receptor, it is suggested that the endothelium of the capillary vessels in the peripheral areas, including hand and foot areas are easily pressurized by daily use. ${ }^{4,5}$ However, no studies to clarify the mechanism of SOR-induced HFS have been reported; therefore, we have focused on the decrease in the blood flow in hand and foot areas whether it can be related to the development of HFS. Also in terms of treatment for HFS, cessation of medication, skin care, and administration of vitamins have been used $;^{7}$ however, no specific and effective method to prevent HFS has been reported to date.

Bonito broth is a traditional Japanese food used to flavor the Japanese dish, "Washoku", which was added to the Intangible Cultural Heritage list by the United Nations Educational, Scientific and Cultural Organization and has been considered as a healthy food. Interestingly, in terms of its mechanism, it has been reported that the ingestion of bonito broth increases peripheral blood flow in humans, ${ }^{9}$ resulting in an antioxidative effect and an improvement in mood. ${ }^{9,12}$ In addition, an increase in the cerebral blood flow in mice ${ }^{10}$ and rats ${ }^{11}$ has been evidenced in the groups ingesting bonito broth, resulting in a decrease in stroke in hypertensive rats. ${ }^{11}$

Therefore, in this study, we examined the mechanism of HFS by monitoring the blood flow in the peripheral lesions and aimed to develop an effective preventive regimen to improve the prognosis of patients with HCC using bonito broth.

\section{Materials and methods Dried-bonito broth (DBB)}

Bonito (Katsuwonus pelamis) is known as katsuo in Japan, and DBB is easy to obtain and is used to add flavor to food. For this study, the patients in the DBB group ingested $150 \mathrm{~mL}$ of commercially available, highly concentrated rich extract of DBB packs (Honzukuri Ichiban Dashi Kiwami Katsuo) orally, once a day in the evening, every day for the entire period of the study. The control group took normal food, which sometimes (one to two times a week) contained the extract; however, the concentration was $\sim 10$-fold lower than the one contained in the pack. The amount of amino acids contained in $150 \mathrm{~mL}$ of DBB is equal to that included in $250 \mathrm{~g}$ of bonito meat. They continued to take either the DBB pack or extracted concentrated bonito broth made from the dried bonito after being discharged from our hospital.

\section{Study protocol and patients}

The study protocol was approved by the ethics committee and institutional review board of Niigata University School of Medicine (clinical trial authorized by the Niigata University, number 1482, on August 30, 2012) and was registered with the University Hospital Medical Information Network Clinical Trials Registry (UMIN000025937) on January 31,2017 . Written informed consent was obtained from all patients, and the study was conducted in accordance with the ethical guidance of the 1975 Declaration of Helsinki. Patients with HCC scheduled for SOR administration were considered eligible for the study if they fulfilled the following criteria: an Eastern Cooperative Oncology performance status of 0-2 and preserved organ function using indicators within the liver (Child-Pugh grade A or B). Patients with the following features were considered ineligible: dermatological issues and had allergies to fish, bonito, or DBB. After informed consent was obtained, patients were divided into two groups: a study group consisting of patients starting ingestion of DBB 1 week prior to SOR initiation (DBB group) and a control group consisting of patients ingesting no DBB. Peripheral blood flow was examined in all patients upon the initiation of the study and 1, 2, and 3 weeks after the initiation (Figure 1). The occurrence of HFS was carefully monitored by either physicians or patients themselves every day during the period of SOR administration. The antitumor response was evaluated by comparing computed tomography (CT)/magnetic resonance imaging (MRI) images obtained before treatment and within 2 months of treatment.

\section{Blood flow analysis}

The peripheral blood flow was measured by examining timeaveraged mean blood flow velocity (TAMEAN, $\mathrm{cm} / \mathrm{s}$ ) in the palm (superficial palmar arch [SPA]), thumb (digital artery), and sole of the foot (medial plantar artery [MPA]) using a Doppler ultrasound ${ }^{13,14}$ with an angle of $<60^{\circ}$ (LOGIQ E9 and 9L-D Linear Probe; frequency: 2.4-10 MHz, GE Healthcare Bio-Sciences Corp., Piscataway, NJ, USA), thermography (TVS-700ME; Nippon Avionics Co., Ltd., Shinagawa-ku, Tokyo, Japan) of two different points on the bilateral hand and foot, ${ }^{12}$ and laser speckle flowgraphy measurements of relative blood velocity (mean blur rate $[\mathrm{MBR}]$ ) of three different points on the central retinal artery (LSFG-NAVI, Softcare Co., Ltd., Fukutsu, Fukuoka, Japan). The values 


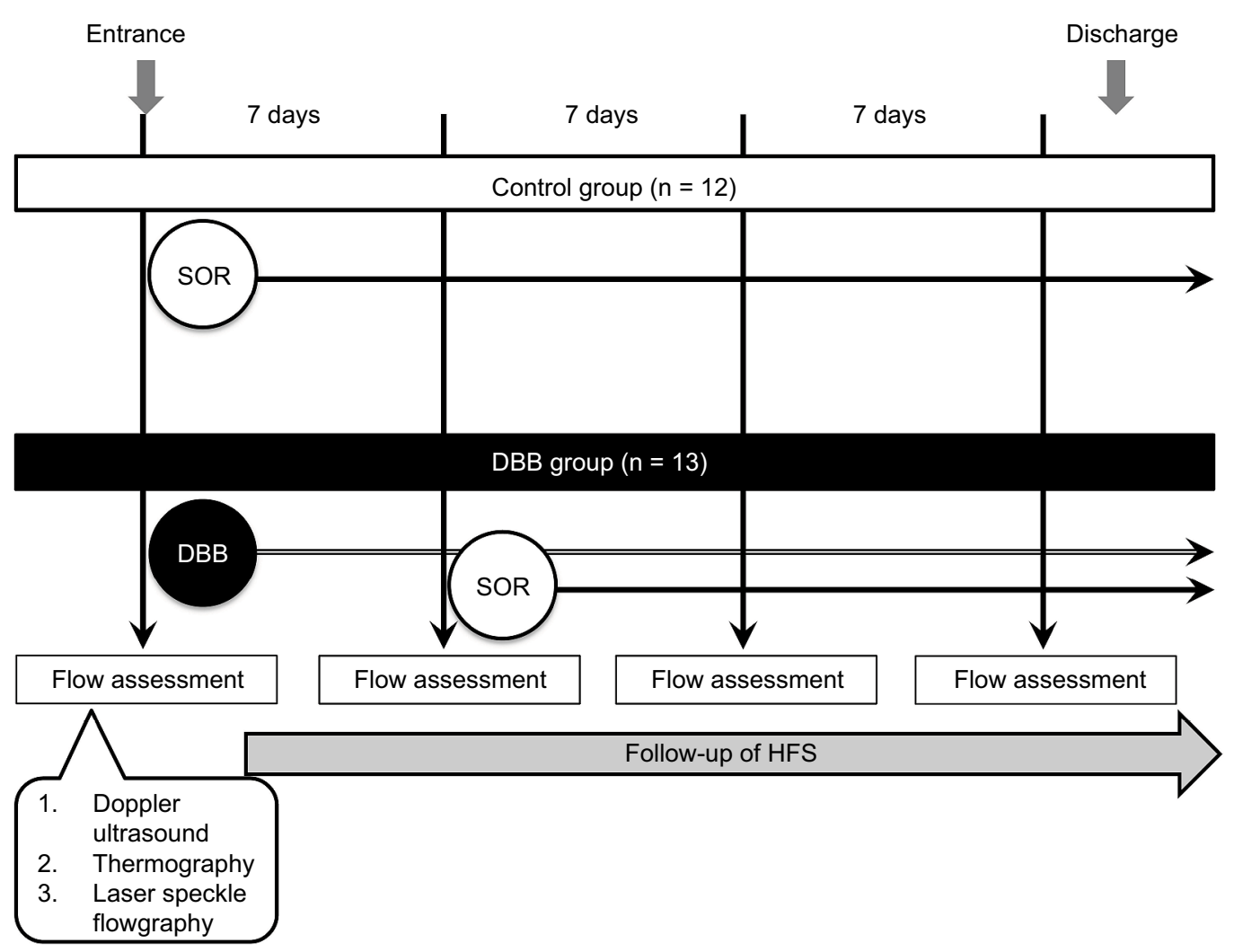

Figure I Study design.

Notes: The control group was treated with SOR without DBB ingestion. The DBB group was treated with SOR with DBB ingestion.

Abbreviations: SOR, sorafenib; DBB, dried-bonito broth; HFS, hand-foot syndrome.

were assessed by comparing the levels of the same modality at each time point. First, three patients from each group were analyzed with all modalities, and based on the results obtained from the three different methods of measuring blood flow, all follow-up evaluations of blood flow were performed by Doppler ultrasound (Figure 2).

\section{Statistical analysis}

Categorical variables were analyzed by the Mann-WhitneyWilcoxon test. The HFS-free survival curve was generated by the Kaplan-Meier method, and the occurrence rate of HFS was compared based on various factors, including age, gender, liver cirrhosis (LC), stage of disease, dose of SOR, tumor controllability, change in peripheral blood flow, and administration of DBB, using a log-rank test. The patients who died without previous HFS detection were censored. The Cox proportional hazards model was used for multivariable analysis of factors that reached statistical significance $(p<0.05)$ in the univariate analyses. Data were analyzed using the Statistical Package for the Social Sciences (SPSS) version 21.0 (IBM Corporation, Armonk, NY, USA). Two-tailed probability values of $p<0.05$ were considered as significant.

\section{Results}

\section{Patient characteristics}

Between 2011 and 2015, a total of 25 patients were screened for eligibility, enrolled in this study, and assigned to either the control group or the DBB group. The baseline characteristics of the patients showed no statistical differences between the two arms for age, gender, etiology of liver disease, presence of LC, Child-Pugh score, stage of HCC, level of alpha-fetoprotein (AFP), dosage of SOR, complication of hypertension (HT), and complication of diabetes mellitus (DM; Table 1).

\section{Effect of peripheral blood flow on HFS}

To examine the effect of peripheral blood flow on the occurrence of HFS, blood flow was assessed by Doppler ultrasound, thermography, and laser speckle flowgraphy (Figure 2). TAMEAN (cm/s) was measured in the bilateral SPA, digital artery, and MPA by Doppler ultrasound (Figure 2A). Temperature was measured by thermography in the bilateral hand and foot at each of the two points (Figure 2B). The MBRs in the retinal artery were measured for each of three points in different branches (Figure 2C). The values at each time point were compared with the initial value, and the 
A

Doppler ultrasound
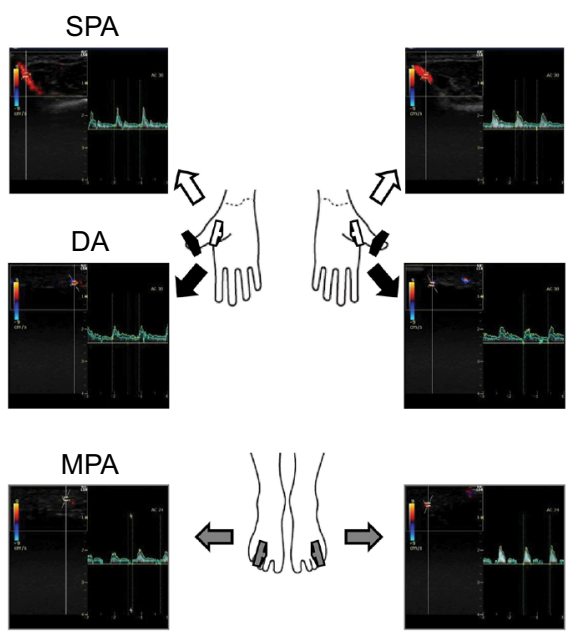

B

Thermography

Before $\quad 1$ week after SOR

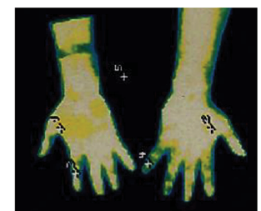

C Laser speckle flowgraphy

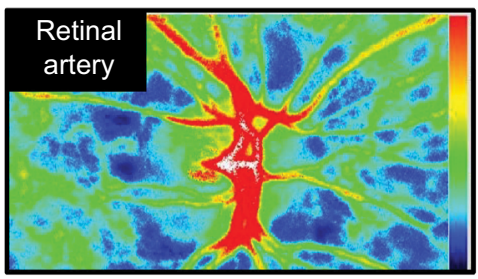

Figure 2 Blood flow analyses.

Notes: (A) Doppler ultrasound for SPA, DA, and MPA. (B) Thermography before and after administration of SOR. (C) Laser speckle flowgraphy to measure the MBR. Abbreviations: SPA, superficial palmar arch; DA, digital artery; MPA, medial plantar artery; SOR, sorafenib; MBR, mean blur rate.

Table I Patient characteristics

\begin{tabular}{|c|c|c|c|}
\hline \multirow[t]{2}{*}{ Characteristics } & \multirow{2}{*}{$\begin{array}{l}\text { Control } \\
-(n=12)\end{array}$} & \multicolumn{2}{|l|}{ DBB } \\
\hline & & $+(n=13)$ & $\begin{array}{l}\text { Total } \\
(N=25)\end{array}$ \\
\hline \multicolumn{4}{|l|}{ Age (years) } \\
\hline Median & 68.0 & 68.0 & 68 \\
\hline Range & $53-85$ & $57-77$ & $53-85$ \\
\hline \multicolumn{4}{|l|}{ Gender } \\
\hline Female & 5 & 2 & 7 \\
\hline Male & 7 & 11 & 18 \\
\hline \multicolumn{4}{|l|}{ Etiology } \\
\hline HBV infection & 2 & 2 & 4 \\
\hline HCV infection & 8 & 8 & 16 \\
\hline Nonalcoholic steatohepatitis & I & 1 & 2 \\
\hline Alcoholic & 0 & 2 & 2 \\
\hline Autoimmune hepatitis & 0 & 0 & 0 \\
\hline Primary biliary cirrhosis & 0 & 0 & 0 \\
\hline Budd-Chiari Syndrome & I & 0 & I \\
\hline \multicolumn{4}{|l|}{ Cirrhosis } \\
\hline Yes/no & $10 / 2$ & $5 / 8$ & $15 / 10$ \\
\hline \multicolumn{4}{|l|}{ Child-Pugh grade } \\
\hline$A / B / C$ & $9 / 1 / 0$ & $5 / 0 / 0$ & $14 / 1 / / 0$ \\
\hline \multicolumn{4}{|l|}{ Stage } \\
\hline I/II/III/IVA/IVB & $0 / 0 / 2 / 6 / 4$ & $0 / 0 / 6 / 5 / 2$ & $0 / 0 / 8 / 11 / 6$ \\
\hline \multicolumn{4}{|l|}{$\operatorname{AFP}(\mathrm{ng} / \mathrm{mL})$} \\
\hline Median & 268.5 & 60 & 90 \\
\hline Range & $4.4-284000$ & $8-28408$ & $4.4-284000$ \\
\hline \multicolumn{4}{|l|}{ SOR (mg/body) } \\
\hline $400 / 600$ & $5 / 7$ & $3 / 10$ & $8 / 17$ \\
\hline $\begin{array}{l}\text { Complication } \\
\text { (HT on medication) }\end{array}$ & 4 & 6 & 10 \\
\hline $\begin{array}{l}\text { Complication } \\
\text { (DM on medication) }\end{array}$ & I & 3 & 4 \\
\hline
\end{tabular}

Abbreviations: DBB, dried-bonito broth; HBV, hepatitis B virus; HCV, hepatitis $C$ virus; AFP, alpha-fetoprotein; SOR, sorafenib; HT, hypertension; DM, diabetes mellitus. occurrence of HFS was carefully monitored for the duration of the study (Figure 3). Representative changes are shown in Figure $3 \mathrm{~A}-\mathrm{C}$, showing a decrease in the blood flow following SOR administration, evidenced by all the modalities examined. Further quantitative analyses were performed by Doppler ultrasound for the entire duration of the follow-up. Of the 12 patients treated with SOR without DBB ingestion, 10 patients showed a decrease in the peripheral blood flow and seven of them developed HFS (Table 2).

\section{Effects of DBB on peripheral blood flow}

To develop an effective process of maintaining peripheral blood flow, DBB was tested for its effect on the peripheral blood flow. First, three patients were examined using all three modalities to measure the blood flow before starting DBB, 1 week after initiation, and 1 and 2 weeks after the administration of SOR ( 2 and 3 weeks after the initiation of DBB; Figure 1). Thermography and MBR clearly demonstrated improved hand and foot peripheral temperature and blood flow in patients who exhibited increased blood flow (Figure 3D-F). In all the other patients in the study, including controls, blood flow was monitored by Doppler ultrasound measuring TAMEAN for all time points.

The changes in the blood flow were compared to baseline levels for each patient 2 weeks after the administration of SOR and noted as decrease, no change, or increase with blood flow changes of $<10 \%$, within $\pm 10 \%$, and $>10 \%$, respectively. Ten patients in the control group, who received SOR without DBB, showed an average decrease in blood flow of $-16.2 \pm$ $9.03 \%$, and only two patients in this group showed no change 

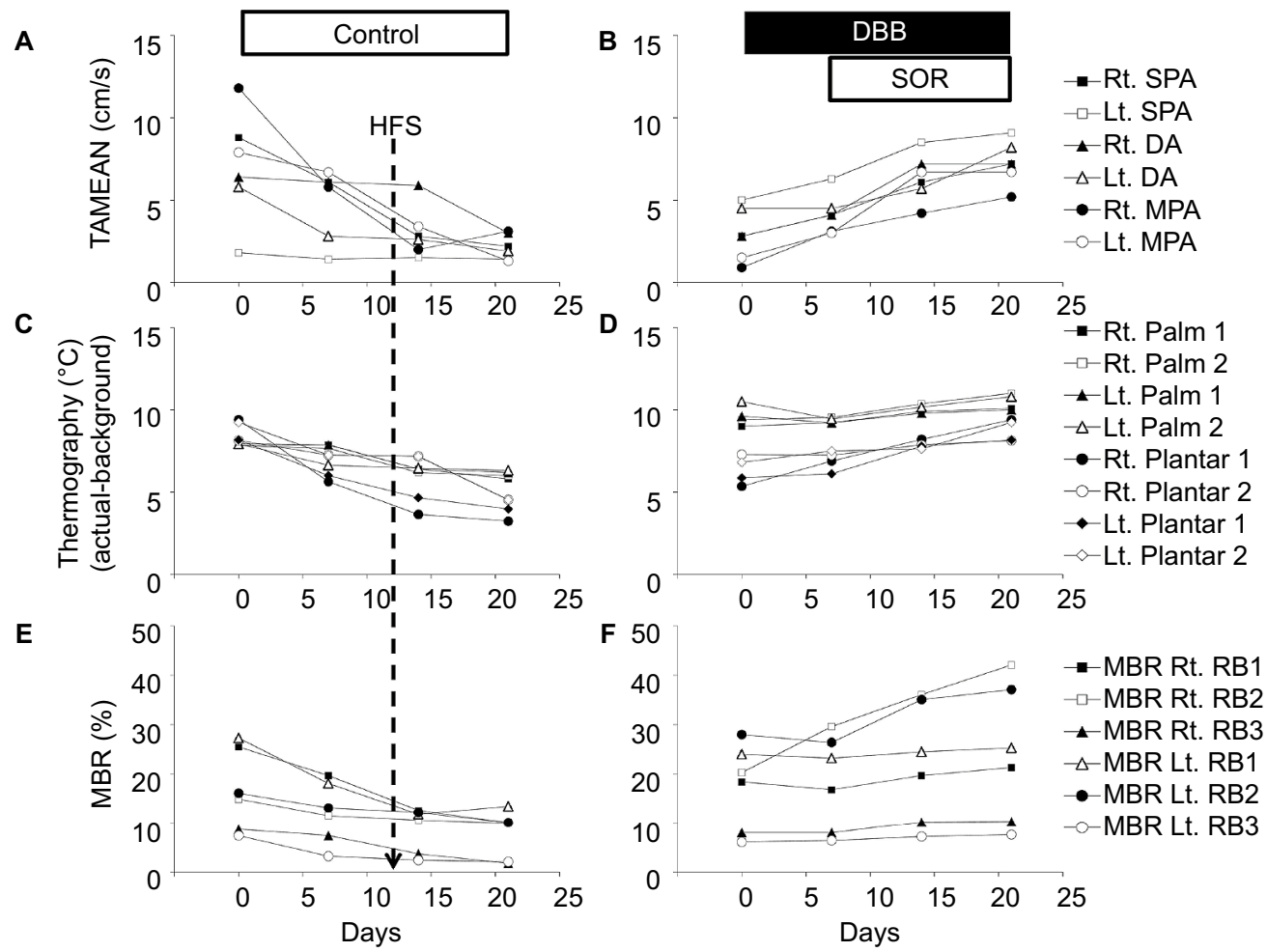

Figure 3 (A and B) Representative time-dependent changes in TAMEAN (cm/s) of bilateral hand and foot. (C and $\mathbf{D})$ Temperature of hand (palm) and foot (plantar) measured by thermography. (E and F) MBR. (A, C, and E) Data of patients in the control group. (B, D, and F) Data of patients in the DBB group.

Abbreviations: TAMEAN, time-averaged mean blood flow velocity; MBR, mean blur rate; DBB, dried-bonito broth; SOR, sorafenib; HFS, hand-foot syndrome; Rt., Right; Lt., Left; SPA, superficial palmar arch; DA, digital artery; MPA, medial plantar artery; RB, retinal blood flow.

Table 2 Summary of patients treated with SOR with or without DBB ingestion

\begin{tabular}{|c|c|c|c|c|}
\hline \multirow[t]{2}{*}{ Summary } & \multirow{2}{*}{$\begin{array}{l}\text { Control } \\
-(n=I 2)\end{array}$} & \multicolumn{3}{|l|}{ DBB } \\
\hline & & $+(n=13)$ & MWW test ( $p$-value) & Total $(\mathbf{N}=\mathbf{2 5})$ \\
\hline Period of observation (days) & & & 0.07 & \\
\hline Median & 46.5 & 160.0 & & 75 \\
\hline Range & $12-822$ & $21-1040$ & & $12-1040$ \\
\hline HFS (n) & 7 & 1 & 0.005 & 8 \\
\hline Blood flow (n) & & & 0.009 & \\
\hline No change (within $\pm 10 \%$ ) & 2 & 2 & & 3 \\
\hline Increase $(>10 \%)$ & 0 & 10 & & 10 \\
\hline Decrease $(<10 \%)$ & 10 & 1 & & 12 \\
\hline Antitumor response $(n)$ & & & 0.38 & \\
\hline $\mathrm{CR} / \mathrm{PR} / \mathrm{SD}$ & 5 & 8 & & 13 \\
\hline PD & 7 & 5 & & 12 \\
\hline DCR (\%) & 41.7 & 61.5 & & 52.0 \\
\hline Progression of HT (n) & 5 & 2 & 0.17 & 7 \\
\hline
\end{tabular}

Abbreviations: SOR, sorafenib; DBB, dried-bonito broth; MWW, Mann-Whitney-Wilcoxon; HFS, hand-foot syndrome; CR, complete response; PR, partial response; SD, stable disease; PD, progressive disease; DCR, disease control rate; HT, hypertension.

in the flow $(3.9 \%$ and $10.0 \%)$. However, 10 patients in the DBB group showed an average increase in the blood flow of $26.8 \pm 18.8 \%$, and only three patients showed a decreased pattern with $-3.1 \%,-3.4 \%$, and $-5.7 \%$. The differences in blood flow (TAMEAN) between the control group and the
DBB group were statistically significant $(p=0.009)$. Over the course of observation, no side effects or progression of HT due to DBB were noted ( $p=0.17)$, and no significant differences in antitumor response $(p=0.38)$ were seen in the two groups (Table 2). 


\section{Effect of DBB on HFS}

The occurrence of HFS was monitored for a median duration of 46.5 days (12-822 days) and 160.0 days (21-1040 days) in the control and DBB groups, respectively (Table 2). The differences in the duration of the follow-up period were due to disease progression and termination of SOR administration, mainly in the control group, due to the side effects.

During this period, eight patients developed HFS, seven in the control group and one in the DBB group $(p=0.005)$, and all showed a decrease in the peripheral blood flow (Table 2). In the control group, four patients had grade $1 \mathrm{HFS}$, one patient had grade 2 HFS, and two patients had grade 3 HFS; the patient in the DBB group had grade 1 HFS.

\section{HFS-free survival}

The factors in patients contributing to the occurrence of HFS were statistically analyzed. First, univariate analyses were performed by the log-rank-test on age ( $\leq 68$ vs. $>68$ years), gender, cirrhosis, HCC stage, SOR dose, antitumor response (complete response, partial response, stable disease vs. progressive disease), change in the blood flow (no change, increase vs. decrease), ingestion of DBB, complications of HT, and complications of DM with the occurrence of HFS as an end point (Table 3).

Gender had a significant effect on HFS-free survival period; a median HFS-free survival of 99 days (14-1040 days) was shown in males vs. 21 days (10-669 days) in females ( $p=0.03$; Figure 4A). Patients with LC showed a significantly shorter HFS-free survival of 28 days (10-1040 days) compared to 221.5 days (14-669 days) in chronic hepatitis patients $(p=0.03$; Figure 4B). The HFS-free survival in the DBB group was 160 days (14-1040 days) vs. 26 days (10-233 days) in the control group, suggesting that DBB significantly contributes to prolong HFS-free period ( $p=0.004$; Figure 4C). The change in the blood flow was also related to the occurrence of HFS. Patients with decreased blood flow showed median HFS-free survival of 22.5 days (14-65 days), while patients with maintained or increased blood flow showed an HFS-free survival of 210 days (101040 days; $p=0.002$; Figure 4D). Multivariable analysis by the Cox proportional hazards model of these four factors showing significance by univariate analysis, and the hazard ratio, 95\% confidence interval, and results of statistical analyses are summarized in Table 3. The ingestion of DBB was a significant independent contributor to HFS-free survival $(p=0.035$; Table 3).

\section{Discussion}

HFS occurs as a side effect of various chemotherapies. ${ }^{7}$ While HFS development due to fluoropyrimidine use is known to be caused by the excretion of drug metabolites through the eccrine sweat glands, the mechanism

Table 3 Univariate and multivariate analyses contributing to HFS-free survival

\begin{tabular}{|c|c|c|c|c|c|c|}
\hline \multirow[t]{2}{*}{ Variable } & \multicolumn{3}{|c|}{ Univariate analysis } & \multicolumn{3}{|c|}{ Multivariable analysis } \\
\hline & Hazard ratio & $95 \% \mathrm{Cl}$ & $p$-value ${ }^{a}$ & Hazard ratio & $95 \% \mathrm{Cl}$ & $p$-value ${ }^{b}$ \\
\hline \multicolumn{7}{|l|}{ Age (years) } \\
\hline$\leq 68$ vs. $>68$ & 2.343 & $0.548-10.01$ & 0.25 & & & \\
\hline \multicolumn{7}{|l|}{ Gender } \\
\hline Female vs. male & 0.133 & $0.022-0.816$ & 0.03 & 0.278 & $0.047-1.631$ & 0.156 \\
\hline \multicolumn{7}{|l|}{ Cirrhosis } \\
\hline Yes vs. no & 0.185 & $0.042-0.812$ & 0.03 & 0.805 & $0.076-8.546$ & 0.857 \\
\hline \multicolumn{7}{|l|}{ Stage } \\
\hline III vs. IVA/IVB & 0.348 & $0.076-1.597$ & 0.17 & & & \\
\hline \multicolumn{7}{|l|}{ SOR (mg/body) } \\
\hline 400 vs. 600 & 0.472 & $0.103-2.147$ & 0.33 & & & \\
\hline \multicolumn{7}{|l|}{ Antitumor response (n) } \\
\hline CR/PR/SD vs. PD & 0.890 & $0.210-3.760$ & 0.87 & & & \\
\hline \multicolumn{7}{|l|}{ Blood flow (n) } \\
\hline No change/increase vs. decrease & 15.48 & $2.70 \mathrm{I}-88.73$ & 0.002 & 0.137 & $0.001-4.047$ & 0.137 \\
\hline \multicolumn{7}{|l|}{ DBB } \\
\hline Yes vs. no & 8.664 & $1.963-38.25$ & 0.004 & 0.097 & $0.011-0.846$ & 0.035 \\
\hline \multicolumn{7}{|l|}{ HT } \\
\hline Yes vs. no & 2.066 & $0.482-8.863$ & 0.33 & & & \\
\hline \multicolumn{7}{|l|}{ DM } \\
\hline Yes vs. no & 3.628 & $0.528-24.93$ & 0.19 & & & \\
\hline
\end{tabular}

Notes: a'Log-rank test. 'bikelihood-ratio test.

Abbreviations: HFS, hand-foot syndrome; Cl, confidence interval; SOR, sorafenib; CR, complete response; PR, partial response; SD, stable disease; PD, progressive disease; DBB, dried-bonito broth; HT, hypertension; DM, diabetes mellitus. 
A

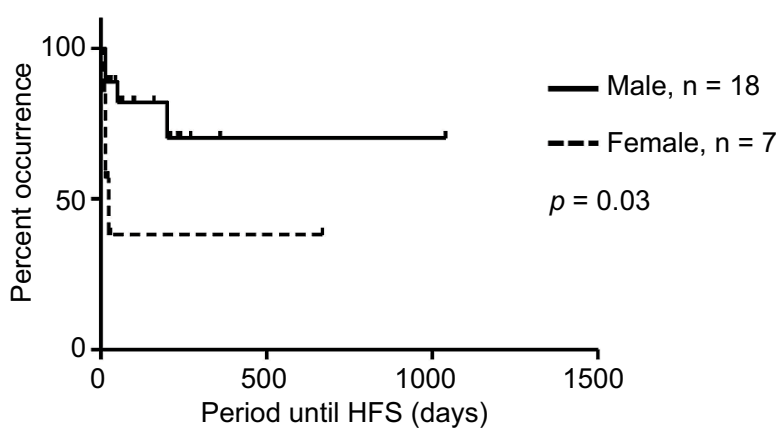

B
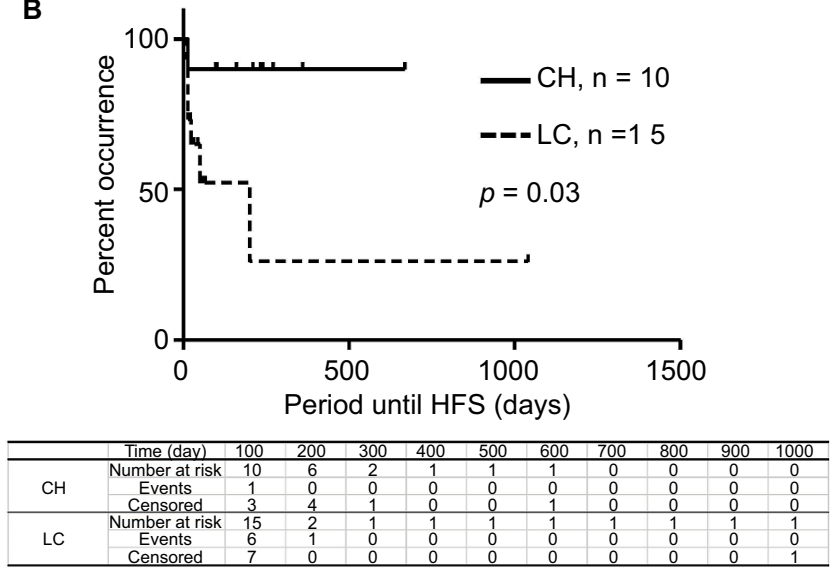

C

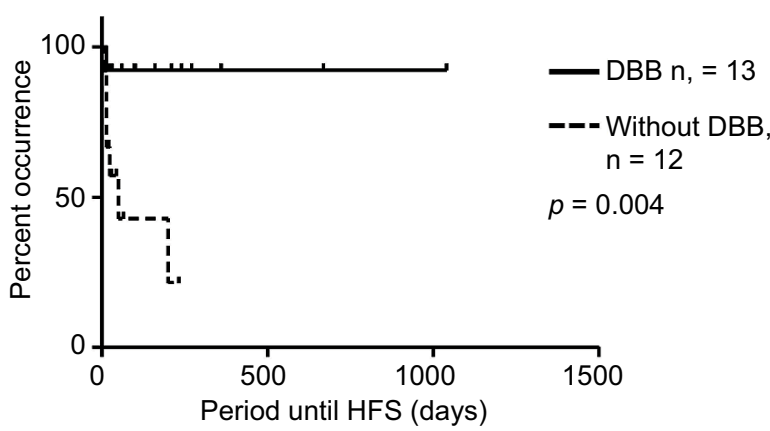

\begin{tabular}{cccccccccccc}
\hline & Time (dav) & 100 & 200 & 300 & 400 & 500 & 600 & 700 & 800 & 900 & 1000 \\
\hline \multirow{2}{*}{ Male } & Number at risk & 13 & 6 & 3 & 2 & 2 & 2 & 1 & 1 & 1 & 1 \\
& Events & 1 & 0 & 0 & 0 & 0 & 0 & 0 & 0 & 0 & 0 \\
& Censored & 6 & 3 & 1 & 0 & 0 & 1 & 0 & 0 & 0 & 1 \\
\hline \multirow{2}{*}{ Female } & Number at risk & 12 & 2 & 0 & 0 & 0 & 0 & 0 & 0 & 0 & 0 \\
& Events & 6 & 1 & 0 & 0 & 0 & 0 & 0 & 0 & 0 & 0 \\
& Censored & 4 & 1 & 0 & 0 & 0 & 0 & 0 & 0 & 0 & 0 \\
\hline
\end{tabular}

D

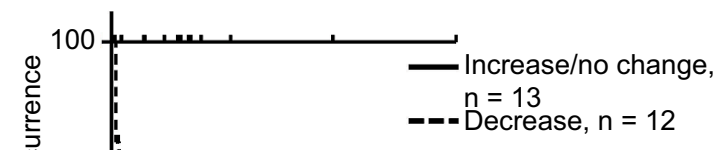

Figure 4 The HFS-free survival curve generated by the Kaplan-Meier method.

Note: The occurrence rate of HFS was compared based on (A) gender, (B) LC or CH, (C) DBB ingestion, (D) change in the peripheral blood flow.

Abbreviations: HFS, hand-foot syndrome; LC, liver cirrhosis; DBB, dried-bonito broth; $\mathrm{CH}$, chronic hepatitis.

of SOR-induced HFS has not yet been reported. ${ }^{7}$ Since SOR contributes to prolong the survival in patients with advanced $\mathrm{HCC},{ }^{1-3}$ it is important to assess the mechanism of HFS development and to discover an effective process to prevent its occurrence. However, it may also damage the peripheral vessels in the hands and feet, leading to an increase in the vascular resistance and a decrease in the blood flow. ${ }^{8}$ This, in turn, increases the blood pressure, leading to sensory disturbance, redness, and sometimes erosions and ulcers that affect the QoL of the patients. ${ }^{4,5}$ Interestingly, while the SHARP trial showed an HFS occurrence ratio of $21.2 \%$ in Caucasians, ${ }^{1}$ an Asia-Pacific trial and a Phase I clinical trial in Japan showed HFS development in $45 \%^{2}$ and $44.4 \%^{3}$ of the patients, respectively. This difference based on race has been understood to be due to a polymorphism of vascular endothelial growth factor. ${ }^{6}$ Based on this information, we hypothesized that the increase in vascular resistance and decreased blood flow might be a potential cause of HFS in patients taking SOR, and we measured the peripheral blood flow in all patients in our study by Doppler ultrasound, thermography, and laser speckle flowgraphy.

Our results clearly demonstrated 1) a decrease in peripheral blood flow after SOR administration (Figure 3); 2) a statistically significant relationship between HFS occurrence and the decrease in peripheral blood flow (Table 3); 3) that all tested modalities were effective for detection of peripheral blood flow and showed consistent results (Figure 2); and 4) that the maintenance of blood flow is the key to prevent occurrence of HFS (Table 2 and Figure 3). To maintain the peripheral blood flow, we have focused on the effect of DBB ingestion on blood flow recovery ${ }^{9-12}$ and performed this clinical trial for the development of effective preventive method for SOR-induced HFS. In terms of effects of DBB on the health, DBB ingestion has been reported to increase significantly the peripheral blood flow in the hands compared to a placebo group. ${ }^{12}$ In addition, Honda et $\mathrm{al}^{11}$ reported that when DBB is administered to 
a rat model of HT, which experiences cerebral stroke with a high frequency, blood pressure elevation was suppressed and cerebral blood flow increased, leading to a significantly lower incidence of cerebrovascular events. Consistent with these results, our patients showed increased blood flow after DBB administration (Figure 3 ) and a significant preventive effect on the development of HFS with an incidence of $7.6 \%$ in patients in the DBB group, which was significantly lower than that of the control group (58.3\%) and the average occurrence of 40-50\% reported in Asian countries by others $^{2,3}$ (Table 2). The multivariable analyses showed a significant contribution of DBB ingestion on the prevention of HFS (Table 3 ). The blood flow showed significance in univariate analysis $(p=0.002)$ but not in multivariable analysis, probably due to the small sample size. In addition, while five in 12 patients showed progression of HT in the control group $(41.7 \%)$, only two in 13 patients $(15.3 \%)$ showed progression in the DBB group, although this difference was not statistically significant. This result also supports the evidence that DBB has an effect on vasodilatation and blood flow recovery. Therefore, we concluded that DBB is effective for maintaining the peripheral blood flow and preventing HFS induced by SOR treatment. It is also noteworthy that no differences in tumor response were seen between the DBB group and the control group. Therefore, we speculate that the efficacy of DBB on SOR-induced HFS may include the vasodilatation of the normal peripheral vessels but not for the tumor arteries. In addition, the various amino acids contained in the $\mathrm{DBB}^{12,15}$ cause this phenomenon. Interestingly, DBB has also been reported to improve fatigue, ${ }^{15}$ mood, ${ }^{16}$ and cognitive function ${ }^{17}$ and has antioxidative properties, ${ }^{18}$ and it might have contributed to maintain the patients' general condition and prolong SOR treatment period. The limitation of our study is that it was designed as an exploratory and pragmatic clinical trial and has several methodological deficiencies with major group imbalances.

\section{Implications for practice}

SOR is a molecular medicine for HCC and has been reported to prolong survival. Therefore, the management of the side effects is essential. Among various side effects, HFS is the most commonly reported, occurring in 30\%-50\% of patients; however, no effective method of its prevention has been reported to date. We report for the first time, in this study, that the mechanism of SOR-induced HFS in the patients with $\mathrm{HCC}$ involves a decrease in the peripheral blood flow and the ingestion of DBB maintains the blood flow significantly, preventing the symptom.

\section{Conclusion}

We have shown that the mechanism of SOR-induced HFS includes a decrease in the peripheral blood flow and the recovery and maintenance of blood flow by DBB contributes to prevent the development of HFS. As DBB is edible and easy to ingest, either by itself or mixing into the food as traditional Japanese food, this study showed the importance of a balanced healthy diet, which leads to a healthy body. Further studies are necessary to clarify the mechanisms of these effects, to determine the active ingredient in DBB, and to examine the amount of either DBB or its ingredients needed to be ingested. Furthermore, additional trial with a larger size and prospectively justified sample is definitely required before any recommendations for the general practice can be given.

\section{Acknowledgment}

The authors would like to thank the Mishima Kaiun Memorial Foundation for the financial support, which, in part, supported the laboratory research.

\section{Author contributions}

$\mathrm{KK}$ and ST were involved in conception and design. YSK, $\mathrm{KO}, \mathrm{TY}, \mathrm{RG}, \mathrm{HB}, \mathrm{TT}, \mathrm{YN}$, and NK contributed to collection and/or assembly of data. AS, SA, TS, MT, HK, SY, KK, YA, and ST carried out data analysis and interpretation. KK and ST performed manuscript writing. All authors contributed to to conception, design, acquisition of data, analysis, or interpretation of data. They drafted the article or revising it critically for important intellectual content

\section{Disclosure}

The authors report no conflicts of interest in this work.

\section{References}

1. Llovet JM, Ricci S, Mazzaferro V, et al. Sorafenib in advanced hepatocellular carcinoma. $N$ Engl J Med. 2008;359(4):378-390.

2. Cheng AL, Kang YK, Chen Z, et al. Efficacy and safety of sorafenib in patients in the Asia-Pacific region with advanced hepatocellular carcinoma: a phase III randomised, double-blind, placebo-controlled trial. Lancet Oncol. 2009;10(1):25-34.

3. Furuse J, Ishii H, Nakachi K, Suzuki E, Shimizu S, Nakajima K. Phase I study of sorafenib in Japanese patients with hepatocellular carcinoma. Cancer Sci. 2008;99(1):159-165.

4. Iijima M, Fukino K, Adachi M, et al. Sorafenib-associated hand-foot syndrome in Japanese patients. J Dermatol. 2011;38(3):261-266.

5. Lacouture ME, Reilly LM, Gerami P, Guitart J. Hand foot skin reaction in cancer patients treated with the multikinase inhibitors sorafenib and sunitinib. Ann Oncol. 2008;19(11):1955-1961.

6. Lee JH, Chung YH, Kim JA, et al. Genetic predisposition of hand-foot skin reaction after sorafenib therapy in patients with hepatocellular carcinoma. Cancer. 2013;119(1):136-142.

7. Macedo LT, Lima JP, dos Santos LV, Sasse AD. Prevention strategies for chemotherapy-induced hand-foot syndrome: a systematic review and meta-analysis of prospective randomised trials. Support Care Cancer. 2014;22(6):1585-1593. 
8. Ishak RS, Aad SA, Kyei A, Farhat FS. Cutaneous manifestations of antiangiogenic therapy in oncology: review with focus on VEGF inhibitors. Crit Rev Oncol Hematol. 2014;90(2):152-164.

9. Nozawa Y, Ishizaki T, Kuroda M, Noguchi T. Effect of dried-bonito broth intake on peripheral blood flow, mood, and oxidative stress marker in humans. Physiol Behav. 2008;93(1-2):267-273.

10. Honda M, Nozawa Y, Ishizaki T, Kuroda M. Ingestion of bonito extract ameliorates peripheral blood flow in mice loaded from over crowding stress. Biomed Res. 2009;30(2):129-135.

11. Honda M, Yamada H, Nozawa Y, Ishizaki T, Kuroda M, Noguchi T. Consumption of bonito extract suppresses the decrease in cerebral blood flow in strokeprone spontaneously hypertensive rats. Biomed Res. 2010;31(4):251-258.

12. Nozawa Y, Kuroda M, Takanori N. Consumption of dried-bonito broth acutely increases peripheral blood flow in humans. J Health Sci. 2007;53:339-343.

13. Kanavaki A, Kattamis A, Delaporta P, et al. Evaluation of intracranial cerebral blood flow velocities in splenectomised and non-splenectomised patients with beta-thalassemia intermedia using transcranial Doppler sonography. In Vivo. 2015;29:501-504.
14. Louis D, Patil S, Saini SS, Kumar P. A Doppler velocimetry evaluation of intestinal blood flow characteristics in neonates receiving intravenous immunoglobulin therapy: a prospective observational study. Indian $J$ Pediatr. 2015;82(6):553-557.

15. Nozawa Y, Yamada K, Okabe Y, Ishizaki T, Kuroda M. The anti-fatigue effects of the low-molecular-weight fraction of bonito extract in mice. Biol Pharm Bull. 2009;32(3):468-474.

16. Kuroda M, Nozawa Y. Effect of dried-bonito broth on mood states: a pooled analysis of four randomized controlled human trials. Biomed Res. 2008;29(4):175-179.

17. Nozawa Y, Mimura M, Yamada K, Sugita M, Shibakusa T, Koyama N. Dried bonito broth improves cognitive function via the histaminergic system in mice. Biomed Res. 2014;35(5):311-319.

18. Intarasirisawat $\mathrm{R}$, Benjakul $\mathrm{S}$, Visessanguan $\mathrm{W}, \mathrm{Wu}$ J. Antioxidative and functional properties of protein hydrolysate from defatted skipjack (Katsuwonous pelamis) roe. Food Chem. 2012;135(4) 3039-3048.

\section{Cancer Management and Research}

\section{Publish your work in this journal}

Cancer Management and Research is an international, peer-reviewed open access journal focusing on cancer research and the optimal use of preventative and integrated treatment interventions to achieve improved outcomes, enhanced survival and quality of life for the cancer patient. The manuscript management system is completely online and includes

\section{Dovepress}

a very quick and fair peer-review system, which is all easy to use. Visit http://www.dovepress.com/testimonials.php to read real quotes from published authors. 